\title{
Postoperative pulmonary edema secondary to rapid fluid injection into the corporal bodies during surgical correction of penile curvature: a rare consequence of artificial erection
}

\author{
Ethan D. Grober, MD, MEd, FRCSC,; Eyun-Jung Shin, MSc ${ }^{\dagger}$
}

\begin{abstract}
Some experts contend that occlusion of the proximal corpora cavernosal bodies of the penis with a tourniquet or manual compression during induction of an artificial erection distorts the penile anatomy and potentially masks proximal curvatures. The current report highlights a rare, but potentially morbid, case of postoperative pulmonary edema as a consequence of rapid fluid injection into the corporal bodies during surgical correction of penile curvature, in a 48-year-old male with a high-risk cardiac history.
\end{abstract}

Can Urol Assoc J 2010;4(2):E42-E44

\section{Background}

Intra-operatively, artificial erections can be induced pharmacologically by intracavernosal injection of vasodilators or by rapid high-pressure fluid (normal saline) injection with or without compression of the corporal bodies. ${ }^{1}$ Some experts contend that occlusion of the proximal corpora cavernosal bodies of the penis with a tourniquet or manual compression during induction of an artificial erection distorts the penile anatomy and potentially masks proximal curvatures. ${ }^{2-4}$ The current report highlights a rare, but potentially morbid, case of postoperative pulmonary edema and congestive heart failure secondary to rapid fluid injection into the corporal bodies during surgical correction of penile curvature.

\section{Case details}

The patient was a 48-year-old male with Peyronie's disease for 18 months (stable for 8 months) and a dominant leftward curvature of the penis of 48 degrees with a wasting deformity at the level of the plaque. Preoperatively, the patient's estimate of maximum achievable penile rigidity with stimulation was reported at $70 \%$. His Sexual Health Inventory for Men (SHIM) score was 17/25 without medications to support erections. He expressed difficulty with vaginal penetration due to penile angulation, but was able to complete sexual intercourse satisfactorily (Sexual Encounter Profile questions 2 and 3). His past medical history was significant for reactive airway disease/bronchitis, non-insulin dependant diabetes, hypercholesterolemia and coronary artery disease with a myocardial infarction and subsequent 4 vessel coronary artery bypass graft 3 years prior. He was a former cigarette smoker. The patient was treated with oral vitamin E prior to evaluation at our centre. Prior to surgery, he was treated with a combination of L-arginine, pentoxifylline and low-dose phosphodiesterase type 5 (PDE5) inhibitors for about 3 months with limited benefit. We discussed various options with the patient, including intralesional injection of verapamil, surgical penile plication and plaque incision with grafting. The patient elected for plaque incision and grafting with small intestinal submucosa (SIS) (Cook Medical, Bloomington, IN) to preserve penile length at the risk of worsening erectile function. To optimize the patient's surgical course, he was evaluated and risk-stratified by the anesthesia and preoperative teams prior to surgery. His preoperative American Society of Anaesthesia (ASA) physical status was 3 (moderate to severe systemic disease limiting some activity). ${ }^{5}$ The electrocardiogram showed a normal sinus rhythm with occasional premature ventricular contractions and boarderline left ventricular hypertrophy. Echocardiography revealed a grade $1 / 4$ (mild) pattern of diastolic heart failure.

Surgery was performed in March 2008 under general anesthetic. Plaques were incised and grafted at 2 separate sites. A running 4-0 biosyn suture was used to sew each graft (sized $30 \%$ larger than the defect) to the edges of the incised defect in the corporal body. To prevent leaks, we tried to minimize (2 to $3 \mathrm{~mm}$ ) travel between suture bites. Our practice is to induce artificial erections with rapid, high-pressure fluid injection into the corporal bodies with intermittent proximal occlusion at the beginning, end and throughout the case as necessary to document the impact of sequential corrections. During the induction of artificial erections, a series (range 1 to 5 ) of intra-corporal injections using about $80 \mathrm{~mL}$ of normal saline via an $80 \mathrm{cc}$ syringe attached to an 18-gauge butterfly needle are "pushed" 


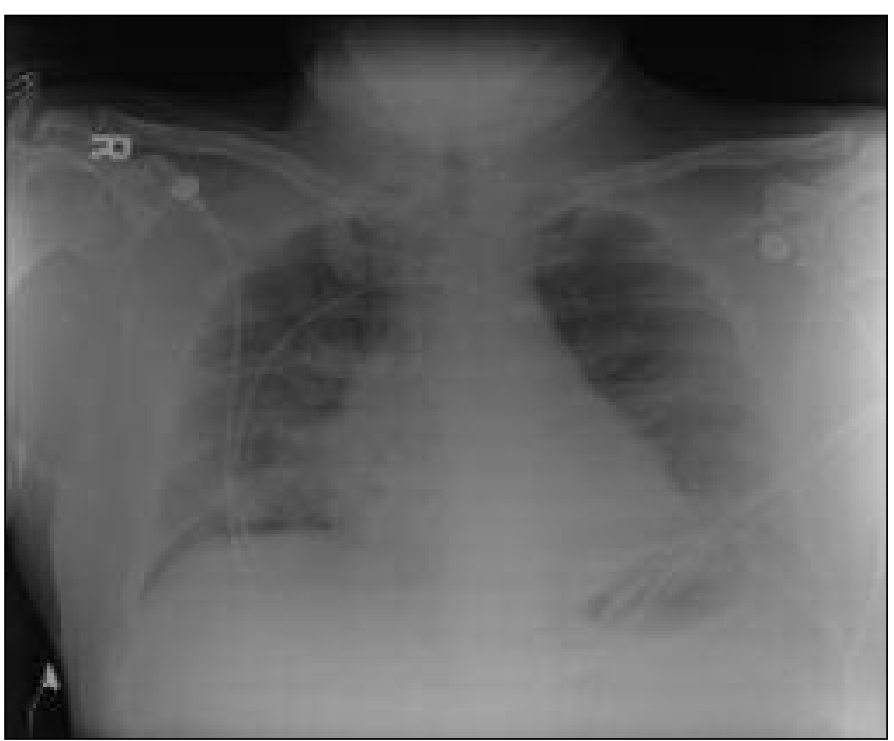

Fig. 1. Immediate postoperative chest radiograph demonstrating pulmonary edema.

rapidly over a few seconds directly into the intracorporal space. This cycle is repeated each time an artificial erection is induced during the case. In total, $800 \mathrm{~mL}$ of normal saline was rapidly injected during this case. Small leaks at the graft sites necessitated prolonged fluid infusion to induce and maintain full erection. In addition, $1000 \mathrm{~mL}$ of ringers lactate was administered intravenously at maintenance levels by the anesthetist during the course of the 150-minute surgery. The operation was completed after a functionally straight penis during peak erection was achieved (less than 15 degrees residual curvature). Vital signs, oxygenation and hemodynamic status were stable during the course of surgery. Immediately postoperatively in the recovery room, the patient appeared tachypneic and complained of shortness of breath. Vital signs revealed a blood pressure of $150 / 83 \mathrm{mmHg}$, heart rate of $104 /$ minute, respiratory rate of $23 /$ minute and oxygen saturation of $80 \%$ on room air. Electrocardiogram demonstrated sinus tachycardia. Chest radiographs highlighted a diffuse air space process consistent with pulmonary edema secondary to congestive heart failure (Fig. 1). Oxygen support was provided by face mask and furosimide was administered intravenously in 2 boluses of $40 \mathrm{mg}$ and $60 \mathrm{mg}$, separated by 30 minutes. Promptly, respiratory signs and symptoms improved and oxygen saturations were maintained over $95 \%$ on room air.

\section{Discussion}

This case highlights the fact that the corporal cavernosal spaces within the penis can essentially act as large calibre venous channels. latrogenic induction of pulmonary edema and congestive heart failure following rapid high-volume fluid injection during artificial erection is possible, particu- larly in high-risk patients with a history of compromised baseline cardiac and respiratory performance. The practice of ongoing communication with members of the anesthesia team on the nature of the penile injections and the potential need for regulation of intravenous fluids is an important lesson of this case.

Intraoperative intracavernosal injection of penile vasodilators should mitigate this complication, but may prove onerous if repeated transitions from tumescent and detumescent states are required throughout the surgery. Moreover, proximal compression of the corporal bodies of the penis facilitates trapping of fluid within the corporal spaces during artificial erection, limiting the volume of fluid required. However, depending on how the forces of pressure are directed, proximal compression can result in unintentional directional and morphological changes to the penis that are unrelated to baseline disease characteristics or the result of surgical correction. For this reason, some experts express caution with this technique fearing it may result in unrecognized persistent/residual curvature..$^{2-4}$ Finally, as illustrated in this report, fluid leaks can occur between suture bites, as well as from suture needle holes within the grafted SIS tissue itself. If the leaks are small, they are typically inconsequential and need not be repaired or can be manually compressed with light pressure during artificial erection. In this case, between-suture leaks made the induction and maintenance of an artificial erection challenging, requiring prolonged fluid infusion to induce and maintain full erection. In retrospect, closure of the leaks would have minimized the volume of fluid delivered.

The preceding example suggests that attention to the volume of fluid injected is warranted and postoperative patient evaluation is prudent, particularly in high-risk patients with a history of compromised cardiac and respiratory performance.

*Assistant Professor, Division of Urology, University of Toronto, Mount Sinai \& Women's College Hospital, Toronto, ON; ${ }^{\top}$ Research Assistant, Division of Urology, University of Toronto, Mount Sinai Hospital, Toronto, ON

\section{Competing interests: None declared.}

This paper has been peer-reviewed.

\section{References}

1. Borer JG, Retik AB. Hypospadias. In: Wein AJ, Kavoussi LR, Novick AC, Partin AW, Peters CA, eds. Campbell-Walsh Urology. 9th ed. Philadelphia, PA: Saunders Elsevier; 2007:3712.

2. Jordan GH. Peyronie's Disease-Surgical Therapy. In: Mulcahy JJ, ed. Male Sexual Function: A Guide to Clinical Management. 2nd ed. Totowa, NJ: Humana Press Inc.; 2006:409.

3. Jordan GH, Rourke KF. Surgical treatment of Peyronie's Disease. In: Graham SD, ed. Glenn's Urologic Surgery. $6^{\text {th }}$ ed. Philadelphia, PA: Lippincott Williams \& Wilkins; 2004:548. 
Grober and Shin

4. Kogan BA. Intraoperative pharmacological erection as an aid to pediatric hypospadias repair. J Urol 2000;164:2058-61.

5. Mak PH, Campbell RC, Irwin MG; American Society of Anesthesiologists. The ASA Physical Status Classification: inter-observer consistency. Anaesth Intensive Care 2002;30:633-40.
Correspondence: Dr. Ethan Grober, Murray Koffler Urologic Wellness Centre, 60 Murray St., 6th Floor, Box 19, Toronto, 0N M5T 3L9; fax: 416-586-8354; egrober@mtsinai.on.ca 